\title{
Categorical characterizations of operator-valued measures
}

\author{
Frank Roumen \\ Inst. for Mathematics, Astrophysics and Particle Physics (IMAPP) \\ Radboud University Nijmegen \\ F.Roumen@math.ru.nl
}

\begin{abstract}
The most general type of measurement in quantum physics is modeled by a positive operator-valued measure (POVM). Mathematically, a POVM is a generalization of a measure, whose values are not real numbers, but positive operators on a Hilbert space. POVMs can equivalently be viewed as maps between effect algebras or as maps between algebras for the Giry monad. We will show that this equivalence is an instance of a duality between two categories. In the special case of continuous POVMs, we obtain two equivalent representations in terms of morphisms between von Neumann algebras.
\end{abstract}

\section{Introduction}

The logic governing quantum measurements differs from classical logic, and it is still unknown which mathematical structure is the best description of quantum logic. The first attempt for such a logic was discussed in the famous paper [2], in which Birkhoff and von Neumann propose to use the orthomodular lattice of projections on a Hilbert space. However, this approach has been criticized for its lack of generality, see for instance [22] for an overview of experiments that do not fit in the Birkhoff-von Neumann scheme. The operational approach to quantum physics generalizes the approach based on projective measurements. In this approach, all measurements should be formulated in terms of the outcome statistics of experiments. Thus the logical and probabilistic aspects of quantum mechanics are combined into a unified description.

The basic concept of operational quantum mechanics is an effect on a Hilbert space, which is a positive operator lying below the identity. It can be viewed as a probabilistic version of a projection. The logical interpretation of an effect is a predicate, or equivalently, a measurement with two possible results. The logic of effects is useful in describing the semantics of quantum programs via weakest preconditions, as argued in [4]. A more general treatment of the logical aspects of effects is given in [15]. Both references use a duality between effects and convex sets to relate syntax and semantics of the logic.

More generally, measurements with an arbitrary space of results can be modeled as maps from the outcome space to the set of effects on a Hilbert space. These maps are called positive operator-valued measures, or POVMs. This paper presents several equivalent characterizations of POVMs, some of them well-known, and some of them new. The results generalize the duality between effects and convex sets. Thus they give a foundation for the connection between syntax and semantics for a quantum logic where the predicates are multivalued instead of two-valued.

The outline of this paper is as follows. Section 2 contains preliminaries about effect algebras, measure theory, and duality between $\sigma$-effect modules and algebras for the Giry monad. This is applied in Section 3 to obtain a categorical rephrasing of the equivalence between POVMs and statistical maps. This result is already known in the literature, but our systematic use of the abstract duality puts it in a broader perspective. In Section 4 we will generalize the sequential composition operation on effects to

M.J. Hoban, B. Coecke (Eds.): 10th International Workshop on Quantum Physics and Logic (QPL 2013) EPTCS 171, 2014, pp. 132-144 doi 10.4204/EPTCS.171.12 (c) Frank Roumen

This work is licensed under the Creative Commons Attribution License. 
POVMs. It will turn out that this only works for a certain class of POVMs, namely those that are differentiable with respect to an ambient measure. This gives a motivation to study these differentiable POVMs in Section 5. To obtain a duality result for differentiable POVMs, we will view them as morphisms between von Neumann algebras.

\section{Preliminaries}

An effect algebra consists of a set $X$ equipped with a partial binary operation $\oplus$, a unary operation $(-)^{\perp}$ called orthocomplement, and a constant $0 \in X$, subject to the following conditions:

- The operation $\oplus$ is commutative, which means that whenever $x \oplus y$ is defined, also $y \oplus x$ is defined and $y \oplus x=x \oplus y$.

- The operation $\oplus$ is associative, defined in a similar way.

- $x \oplus 0=0 \oplus x=x$ for all $x \in X$.

- For every $x \in X, x^{\perp}$ is the unique element for which $x \oplus x^{\perp}=1$, where 1 is defined as $0^{\perp}$.

- If $x \oplus 1$ is defined, then $x=0$.

Effect algebras constitute a category EA, in which the morphisms are functions preserving $\oplus,(-)^{\perp}$, and 0 . Effect algebras originated in the study of quantum logics in [7], and can be used to describe both the probabilistic and the logical aspects of quantum mechanics. An overview of the theory of effect algebras is given in [6].

The principal example of an effect algebra is the unit interval $[0,1]$. Addition serves as a partially defined binary operation, and the orthocomplement is given by $x^{\perp}=1-x$. Another important example comes from quantum logic. An effect on a Hilbert space $H$ is an operator $A: H \rightarrow H$ for which $0 \leq A \leq \mathrm{id}$. The set $\mathscr{E} f(H)$ of all effects on $H$ forms an effect algebra, in which the partial binary operation is again addition, and orthocomplement is $A^{\perp}=\mathrm{id}-A$. Furthermore each Boolean algebra $B$ can be viewed as an effect algebra, where $x \oplus y$ is defined if and only if $x \wedge y=0$, and in that case $x \oplus y=x \vee y$. The orthocomplement is simply the complement in $B$.

Some effect algebras carry additional structure, which leads to several commonly used subcategories of EA. First we will consider the subcategory EMod of effect modules. An effect module is an effect algebra $X$ endowed with a scalar multiplication $\cdot:[0,1] \times X \rightarrow X$, such that

- $r \cdot(s \cdot x)=(r s) \cdot x$.

- If $r+s \leq 1$, then $(r+s) \cdot x=r \cdot x \oplus s \cdot x$.

- If $x \oplus y$ is defined, then $r \cdot(x \oplus y)=r \cdot x \oplus r \cdot y$.

- $1 \cdot x=x$.

Effect modules were introduced in [13] under the name 'convex effect algebras', and generalized in [18] to modules over arbitrary effect algebras with a monoid structure, rather than just over the interval $[0,1]$. Morphisms of effect modules are morphisms of effect algebras that additionally preserve the scalar multiplication. From our three examples of effect algebras, only $[0,1]$ and $\mathscr{E} f(H)$ are effect modules.

If $X$ is any effect algebra, then we can define a partial order on $X$ by setting $x \leq y$ if and only if $x \oplus z=y$ for some $z \in X$. The algebra $X$ is said to be an $\sigma$-effect algebra if each countable chain in $X$ has a join in $X$. This gives rise to a subcategory $\sigma \mathbf{E A}$ of EA in which the morphisms also preserve joins of countable chains. A $\sigma$-effect algebra that is at the same time an effect module is called a $\sigma$-effect module, 
and they constitute a category $\sigma$ EMod. The unit interval and $\mathscr{E} f(H)$ are always $\sigma$-effect modules. A Boolean algebra is a $\sigma$-effect algebra if and only if it is a $\sigma$-algebra.

Given two effect algebras $X$ and $Y$, one can form their tensor product $X \otimes Y$ characterizing the bimorphisms out of $X \times Y$. This tensor product can be used to construct free effect modules: for any effect algebra $X$, the tensor product $[0,1] \otimes X$ is the free effect module generated by $X$. The situation is more subtle for $\sigma$-effect algebras, because the tensor product of two $\sigma$-effect algebras need not always exist. This problem is discussed in [9].

Effect algebras also occur in measure theory. A measurable space consists of a set $X$ together with a $\sigma$-algebra of subsets of $X$, denoted $\Sigma_{X}$. Measurable spaces constitute a category Meas, in which the maps from $X$ to $Y$ are functions $f: X \rightarrow Y$ for which $f^{-1}\left(\Sigma_{Y}\right) \subseteq \Sigma_{X}$. As each $\sigma$-algebra is an effect algebra with countable joins, there is a functor $\Sigma_{(-)}$: Meas $\rightarrow \sigma \mathbf{E} \mathbf{A}^{\mathrm{op}}$. The $\sigma$-effect algebra $\Sigma_{X}$ can be turned into a $\sigma$-effect module by taking the tensor product $[0,1] \otimes \Sigma_{X}$. In [9] it is shown that this tensor product exists and is isomorphic to the algebra $\operatorname{Meas}(X,[0,1])$ of measurable functions from $X$ to the unit interval. In other words, $\operatorname{Meas}(X,[0,1])$ is the free $\sigma$-effect module generated by the $\sigma$-algebra $\Sigma_{X}$.

Giry initiated the categorical approach to measure and integration theory in [8] by defining the Giry monad $\mathscr{G}$ on the category Meas as $\mathscr{G}(X)=\sigma \mathbf{E} \mathbf{A}\left(\Sigma_{X},[0,1]\right)$. Thus the elements of $\mathscr{G}(X)$ are probability measures. A measurable map $p: X \rightarrow[0,1]$ can be integrated along a probability measure $\varphi \in \mathscr{G}(X)$ to obtain $\int p \mathrm{~d} \varphi \in[0,1]$, sometimes written as $\int p(x) \mathrm{d} x$ if $\varphi$ is understood.

In [16] it is shown that there is a dual adjunction between Eilenberg-Moore algebras for the Giry monad and $\sigma$-effect modules:

$$
\operatorname{Alg}(\mathscr{G}) \underset{\operatorname{Hom}(-,[0,1])}{\frac{\operatorname{Hom}(-,[0,1])}{\rightleftarrows}} \sigma \mathbf{E M o d}^{\mathrm{op}}
$$

This gives a foundation for probabilistic and quantum logic, since a $\mathscr{G}$-algebra can be considered as the state space of a system, and the corresponding $\sigma$-effect module gives the predicates on that system.

\section{Duality for POVMs}

Effects on a Hilbert space can be seen as yes-no questions about the physical system represented by the Hilbert space. It is also possible to consider more general questions, which have answers lying in an arbitrary measurable space. These can be mathematically modeled by positive operator-valued measures.

Definition 1. Let $\left(X, \Sigma_{X}\right)$ be a measurable space. A positive operator-valued measure (POVM) on $X$ is a morphism $\Sigma_{X} \rightarrow \mathscr{E} f(H)$ of $\sigma$-effect algebras. A POVM is a projection-valued measure $(P V M)$ if its image is contained in $\mathscr{P} \operatorname{roj}(H)$.

We will study these POVMs from the viewpoint of categorical logic. The syntax of a logic is obtained by defining operations on predicates, leading to an algebraic structure. For instance, the predicates in operational quantum logic are effects on a Hilbert space, and the appropriate operations are the $\sigma$-effect algebra operations. The semantics of a logic is related to the syntax via duality. In our quantum example, the semantics is given by density matrices, since density matrices and effects are related via the duality between convex sets and effect algebras, see [17] for details.

In the remainder of this paper, we will try to establish a similar picture for POVMs. This section considers a generalization of the duality for effects to POVMs. The duality for POVMs will be based on the adjunction (1), so it is helpful to rephrase the definition of POVMs in terms of morphisms between modules. 
Lemma 2. There is a bijective correspondence between POVMs $\Sigma_{X} \rightarrow \mathscr{E} f(H)$ and morphisms of $\sigma$-effect modules $\operatorname{Meas}(X,[0,1]) \rightarrow \mathscr{E} f(H)$.

This follows immediately from Gudder's result that $\operatorname{Meas}(X,[0,1])$ is the free $\sigma$-effect module on $\Sigma_{X}$, which was briefly mentioned in Section 2 More explicitly, if $\varphi: \Sigma_{X} \rightarrow \mathscr{E} f(H)$ is a POVM, then the corresponding map Meas $(X,[0,1]) \rightarrow \mathscr{E} f(H)$ is given by integration along the POVM $\varphi$, i.e. $p \mapsto \int p \mathrm{~d} \varphi$. The inverse construction is evaluation at an indicator function, that is, a map $\Phi: \operatorname{Meas}(X,[0,1]) \rightarrow \mathscr{E} f(H)$ gives a POVM $M \mapsto \Phi\left(\mathbf{1}_{M}\right)$.

Lemma 3. There is a functor $\mathscr{D} \mathscr{M}: \mathbf{H i l b}_{\text {isomet }} \rightarrow \mathbf{A l g}(\mathscr{G})$ that maps a Hilbert space $H$ to the set of density matrices on $\mathrm{H}$. Here Hilb $_{\text {isomet }}$ is the category with Hilbert spaces as objects and isometries as morphisms.

Proof. First we have to endow $\mathscr{D} \mathscr{M}(H)$ with the structure of a measurable space. The weak operator topology on $\mathscr{B}(H)$ restricts to a subset topology on $\mathscr{D} \mathscr{M}(H)$. Let $\Sigma_{\mathscr{D} \mathscr{M}(H)}$ be the Borel $\sigma$-algebra generated by the topology on $\mathscr{D} \mathscr{M}(H)$. The resulting measurable space $\left(\mathscr{D} \mathscr{M}(H), \Sigma_{\mathscr{D} \mathscr{M}(H)}\right)$ is an algebra for the Giry monad with algebra map $\alpha: \mathscr{G}(\mathscr{D} \mathscr{M}(H)) \rightarrow \mathscr{D} \mathscr{M}(H), \alpha(\varphi)=\int \mathrm{id} \mathrm{d} \varphi$. Here the integration is defined in such a way that $\left\langle\psi\left|\int \operatorname{idd} \varphi\right| \psi\right\rangle=\int\langle\psi|(-)| \psi\rangle \mathrm{d} \varphi$ for each vector $\psi$. The map $\alpha$ is measurable by general facts about integration. To show that the integral is a density matrix, let $\left(e_{k}\right)$ be an orthonormal basis for $H$. Then

$$
\operatorname{tr}\left(\int \operatorname{idd} \varphi\right)=\sum_{k}\left\langle e_{k}\left|\int \operatorname{idd} \varphi\right| e_{k}\right\rangle=\sum_{k} \int\left\langle e_{k}|(-)| e_{k}\right\rangle \mathrm{d} \varphi=\int \sum_{k}\left\langle e_{k}|(-)| e_{k}\right\rangle \mathrm{d} \varphi=\int 1 \mathrm{~d} \varphi=1 .
$$

Proving that $\alpha$ is an Eilenberg-Moore algebra is straightforward.

If $f: H \rightarrow K$ is an isometry between Hilbert spaces, then $f$ induces a map $\mathscr{D} \mathscr{M}(f): \mathscr{D} \mathscr{M}(H) \rightarrow$ $\mathscr{D} \mathscr{M}(K)$ via conjugation, i.e. $\mathscr{D} \mathscr{M}(f)(\rho)=f \circ \rho \circ f^{\dagger}$. The resulting map is a $\mathscr{G}$-algebra homomorphism. Before proving this, we first remark that conjugation commutes with integration in the sense that $\int\left(f(-) f^{\dagger}\right) \mathrm{d} \varphi=f\left(\int(-) \mathrm{d} \varphi\right) f^{\dagger}$. This follows because for each vector $\psi$ we have

$$
\begin{aligned}
\left\langle\psi\left|\int f(-) f^{\dagger} \mathrm{d} \varphi\right| \psi\right\rangle & =\int\left\langle\psi\left|f(-) f^{\dagger}\right| \psi\right\rangle \mathrm{d} \varphi \\
& =\int\left\langle f^{\dagger} \psi|(-)| f^{\dagger} \psi\right\rangle \mathrm{d} \varphi \\
& =\left\langle f^{\dagger} \psi\left|\int(-) \mathrm{d} \varphi\right| f^{\dagger} \psi\right\rangle \\
& =\left\langle\psi\left|f\left(\int(-) \mathrm{d} \varphi\right) f^{\dagger}\right| \psi\right\rangle
\end{aligned}
$$

Using this we can show that $\mathscr{D} \mathscr{M}(f)$ is a $\mathscr{G}$-algebra homomorphism:

$$
\begin{aligned}
(\alpha \circ \mathscr{G}(\mathscr{D} \mathscr{M}(f)))(\varphi) & =\int \operatorname{idd} \mathscr{G}(\mathscr{D} \mathscr{M}(f))(\varphi) \\
& =\int \mathscr{D} \mathscr{M}(f) \mathrm{d} \varphi \\
& =\int f(-) f^{\dagger} \mathrm{d} \varphi \\
& =f\left(\int(-) \mathrm{d} \varphi\right) f^{\dagger} \\
& =\mathscr{D} \mathscr{M}(f)\left(\int \operatorname{idd} \varphi\right) \\
& =(\mathscr{D} \mathscr{M}(f) \circ \alpha)(\varphi)
\end{aligned}
$$

This shows that $\mathscr{D} \mathscr{M}$ is a well-defined functor.

The collections of density matrices and effects on a Hilbert space are related via the adjunction (1), just like in the discrete probabilistic case.

Proposition 4. Fix a Hilbert space H. Then:

1. The $\mathscr{G}$-algebras $\sigma \mathbf{E M o d}(\mathscr{E} f(H),[0,1])$ and $\mathscr{D} \mathscr{M}(H)$ are isomorphic. 
2. The $\sigma$-effect modules $\mathbf{A} \lg (\mathscr{G})(\mathscr{D} \mathscr{M}(H),[0,1])$ and $\mathscr{E} f(H)$ are isomorphic.

Proof.

1. This is a reformulation of Busch's theorem in [3].

2. In [18] this result is proven for affine maps $\mathscr{D} \mathscr{M}(H) \rightarrow[0,1]$ instead of $\mathscr{G}$-algebra maps, so the statement follows because every $\mathscr{G}$-algebra map is in particular affine.

Since $[0,1] \cong \mathscr{G}(2)$, measurable maps into $[0,1]$ are the same as morphisms into 2 in the Kleisli category $\mathbf{K l}(\mathscr{G})$. The following diagram summarizes the relations between the logic of measurable spaces and the logic of Hilbert spaces.

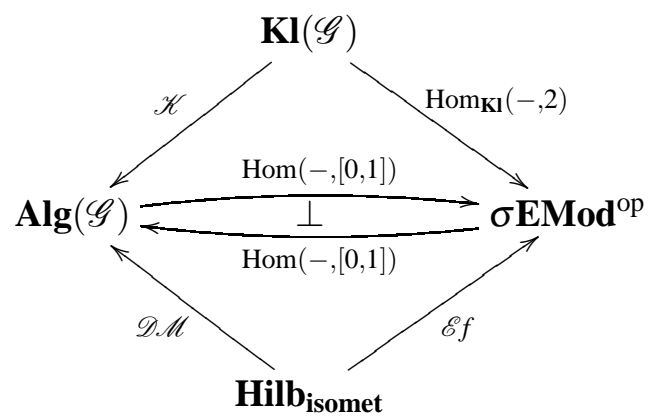

The functor $\mathscr{K}$ is the comparison functor sending an object $X \in \mathbf{K l}(\mathscr{G})$ to the free algebra $\mathscr{G}(X)$. In this setting we can consider the comma categories $(\mathscr{D} \mathscr{M} \downarrow \mathscr{K})$ and $(\mathscr{E} f \downarrow \operatorname{Hom} \mathbf{K I}(-, 2))$. An object of the category $(\mathscr{D} \mathscr{M} \downarrow \mathscr{K})$ is a map of the form $\mathscr{D} \mathscr{M}(H) \rightarrow \mathscr{G}(X)$. A morphism from $\alpha: \mathscr{D} \mathscr{M}(H) \rightarrow \mathscr{G}(X)$ to $\beta: \mathscr{D} \mathscr{M}(K) \rightarrow \mathscr{G}(Y)$ is a commutative diagram

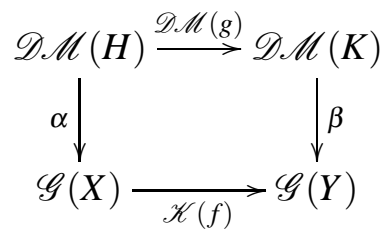

where $f: X \rightarrow \mathscr{G}(Y)$ is a measurable map and $g: H \rightarrow K$ is an isometry. Since the functors $\mathscr{E} f$ and $\operatorname{Hom}_{\mathbf{K I}}(-, 2)$ have the opposite of $\sigma$ EMod as codomain, an object of $\left(\mathscr{E} f \downarrow \operatorname{Hom}_{\mathbf{K I}}(-, 2)\right)$ is a morphism $\operatorname{Hom}_{\mathbf{K I}}(X, 2) \rightarrow \mathscr{E} f(H)$ in $\sigma$ EMod, that is, a POVM. A morphism between two POVMs $A: \operatorname{Hom}_{\mathbf{K l}}(X, 2) \rightarrow \mathscr{E} f(H)$ and $B: \operatorname{Hom}_{\mathbf{K I}}(Y, 2) \rightarrow \mathscr{E} f(K)$ is given by a diagram

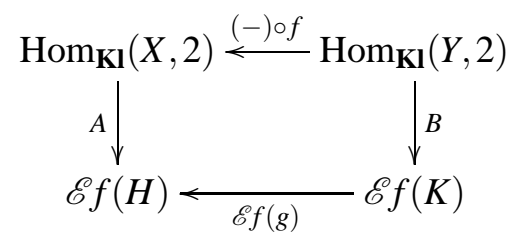

in $\sigma$ EMod, for a measurable map $f: X \rightarrow \mathscr{G}(Y)$ and an isometry $g: H \rightarrow K$.

In [14] it is shown that there is a correspondence between POVMs and $\mathscr{G}$-algebra homomorphisms $\mathscr{D} \mathscr{M}(H) \rightarrow \mathscr{G}(X)$, called statistical maps. From a categorical perspective, this can be phrased as an equivalence between comma categories as follows. 
Proposition 5. The categories $(\mathscr{D} \mathscr{M} \downarrow \mathscr{G})$ and $(\mathscr{E} f \downarrow \operatorname{Hom} \mathbf{K I}(-, 2))$ are equivalent.

Proof. An object of $(\mathscr{D} \mathscr{M} \downarrow \mathscr{G})$ is the same as a morphism $\mathscr{D} \mathscr{M}(H) \rightarrow \sigma \operatorname{EMod}\left(\operatorname{Hom}_{\mathbf{K l}}(X, 2),[0,1]\right)$ since $\operatorname{Hom}_{\mathbf{K I}}(X, 2) \cong \operatorname{Meas}(X,[0,1])$ is the free $\sigma$-effect module on $\Sigma_{X}$. By the adjunction (11) and Proposition 4 this corresponds to a POVM. For morphisms, let $f: X \rightarrow \mathscr{G}(Y)$ be a measurable map, $g: H \rightarrow K$ an isometry, and $\alpha: \mathscr{D} \mathscr{M}(H) \rightarrow \mathscr{G}(X)$ and $\beta: \mathscr{D} \mathscr{M}(K) \rightarrow \mathscr{G}(Y)$ two statistical maps. Then the diagram (2) commutes if and only if the corresponding diagram (3) commutes.

\section{Sequential composition}

Suppose that we want to test two properties of a physical system sequentially. If the properties are modeled by effects $A$ and $B$, then the composite test corresponds to the effect $\sqrt{A} B \sqrt{A}$, which is called the sequential product of $A$ and $B$. The properties of this operation are studied in [12, 11, 10]. We will now define an extension of this operation to POVMs, which can be used if we want to measure two POVMs sequentially. We start by measuring a POVM $A: \Sigma_{X} \rightarrow \mathscr{E} f(H)$. The outcome of this measurement is a value $x \in X$. The second POVM may depend on the outcome of the first measurement, so we assume that we have a family of measurable spaces $\left(Y_{x}\right)$ indexed by $x \in X$ with a family of POVMs $B=\left(B_{x}: \Sigma_{Y_{x}} \rightarrow \mathscr{E} f(H)\right)$. We wish to define a POVM representing the total experiment. For this we need the additional assumptions that the measurable space $X$ is equipped with a finite measure $\mu: \Sigma_{X} \rightarrow \mathbb{R}$, and that $A$ has a Radon-Nikodym derivative with respect to $\mu$. Recall that a Radon-Nikodym derivative of $A$ with respect to $\mu$ is a function $\frac{\mathrm{d} A}{\mathrm{~d} \mu}: X \rightarrow \mathscr{P}_{O S}(H)$ for which $\int_{M} \frac{\mathrm{d} A}{\mathrm{~d} \mu} \mathrm{d} \mu=A(M)$ for each measurable subset $M \subseteq X$. Here $\mathscr{P}_{O S}(H)$ denotes the set of positive operators on $H$. The derivative, if it exists, is unique up to equality almost everywhere. Conditions for existence are discussed in e.g. [5]. Here we will only briefly state the result that we need for the remainder of this paper. The POVM $A$ is called $\mu$-continuous if $A(M)=0$ whenever $\mu(M)=0$. It has bounded variation if

$$
\sup \sum_{i=1}^{n}\left\|\varphi\left(X_{i}\right)\right\|<\infty
$$

where the supremum is taken over all finite partitions $X=\bigcup_{i=1}^{n} X_{i}$ of the space $X$. If the Hilbert space $H$ is finite-dimensional, then the POVM $A$ has a derivative if and only if it is $\mu$-continuous and has bounded variation, because $\mathscr{B}(H)$ has the Radon-Nikodym property.

Under the assumption that $A$ has a Radon-Nikodym derivative $\frac{\mathrm{d} A}{\mathrm{~d} \mu}$, we can define the sequential composition of the POVM $A$ and the family $B$. The total outcome of the experiment consists of a value $x \in X$ together with a value $y \in Y_{x}$, so our outcome space is $Y=\bigcup_{x \in X} Y_{x}$. The union carries a natural $\sigma$-algebra generated by $\bigcup_{x \in M} N_{x}$, where $M$ is a measurable subset of $X$ and each $N_{x}$ is a measurable subset of $Y_{x}$. Define the sequential composition by

$$
\begin{gathered}
(A ; B): \Sigma_{Y} \rightarrow \mathscr{E} f(H) \\
(A ; B)\left(\bigcup_{x \in M} N_{x}\right)=\int_{M}\left(\sqrt{\frac{\mathrm{d} A}{\mathrm{~d} \mu}(x)} B_{x}\left(N_{x}\right) \sqrt{\frac{\mathrm{d} A}{\mathrm{~d} \mu}(x)}\right) \mathrm{d} x
\end{gathered}
$$

Lemma 6. The sequential composition $(A ; B)$ is a POVM. 
Proof. Suppose that the measurable sets $\bigcup_{x \in M} N_{x}$ and $\bigcup_{x \in M^{\prime}} N_{x}^{\prime}$ are disjoint. Then their union can be written as

$$
\bigcup_{x \in M \backslash M^{\prime}} N_{x} \cup \bigcup_{x \in M^{\prime} \backslash M} N_{x}^{\prime} \cup \bigcup_{x \in M \cap M^{\prime}}\left(N_{x} \cup N_{x}^{\prime}\right),
$$

where $N_{x}$ and $N_{x}^{\prime}$ are disjoint whenever both are defined. Applying the map $A ; B$ gives

$$
\begin{aligned}
(A ; B)\left(\bigcup_{x \in M} N_{x} \cup \bigcup_{x \in M^{\prime}} N_{x}^{\prime}\right)= & \int_{M \backslash M^{\prime}} \sqrt{\frac{\mathrm{d} A}{\mathrm{~d} \mu}(x)} B_{x}\left(N_{x}\right) \sqrt{\frac{\mathrm{d} A}{\mathrm{~d} \mu}(x)} \mathrm{d} x \\
& +\int_{M^{\prime} \backslash M} \sqrt{\frac{\mathrm{d} A}{\mathrm{~d} \mu}(x)} B_{x}\left(N_{x}^{\prime}\right) \sqrt{\frac{\mathrm{d} A}{\mathrm{~d} \mu}(x)} \mathrm{d} x \\
& +\int_{M \cap M^{\prime}} \sqrt{\frac{\mathrm{d} A}{\mathrm{~d} \mu}(x)} B_{x}\left(N_{x} \cup N_{x}^{\prime}\right) \sqrt{\frac{\mathrm{d} A}{\mathrm{~d} \mu}(x)} \mathrm{d} x \\
= & \int_{M \backslash M^{\prime}} \sqrt{\frac{\mathrm{d} A}{\mathrm{~d} \mu}(x)} B_{x}\left(N_{x}\right) \sqrt{\frac{\mathrm{d} A}{\mathrm{~d} \mu}(x)} \mathrm{d} x \\
& +\int_{M^{\prime} \backslash M} \sqrt{\frac{\mathrm{d} A}{\mathrm{~d} \mu}(x)} B_{x}\left(N_{x}^{\prime}\right) \sqrt{\frac{\mathrm{d} A}{\mathrm{~d} \mu}(x)} \mathrm{d} x \\
& +\int_{M \cap M^{\prime}} \sqrt{\frac{\mathrm{d} A}{\mathrm{~d} \mu}(x)} B_{x}\left(N_{x}\right) \sqrt{\frac{\mathrm{d} A}{\mathrm{~d} \mu}(x)} \mathrm{d} x \\
& +\int_{M \cap M^{\prime}} \sqrt{\frac{\mathrm{d} A}{\mathrm{~d} \mu}(x)} B_{x}\left(N_{x}^{\prime}\right) \sqrt{\frac{\mathrm{d} A}{\mathrm{~d} \mu}(x)} \mathrm{d} x \\
= & \int_{M} \sqrt{\frac{\mathrm{d} A}{\mathrm{~d} \mu}(x)} B_{x}\left(N_{x}\right) \sqrt{\frac{\mathrm{d} A}{\mathrm{~d} \mu}(x)} \mathrm{d} x \\
& +\int_{M^{\prime}} \sqrt{\frac{\mathrm{d} A}{\mathrm{~d} \mu}(x)} B_{x}\left(N_{x}^{\prime}\right) \sqrt{\frac{\mathrm{d} A}{\mathrm{~d} \mu}(x)} \mathrm{d} x \\
= & (A ; B)\left(\bigcup_{x \in M^{\prime}} N_{x}\right)+(A ; B)\left(\bigcup_{x \in M^{\prime}} N_{x}^{\prime}\right)
\end{aligned}
$$

Hence the map $A ; B$ is additive. It is not hard to check that it preserves the unit. Finally, each operator $(A ; B)\left(\bigcup_{x \in M} N_{x}\right)$ is positive, and lies below the identity because $(A ; B)\left(\bigcup_{x \in M} N_{x}\right) \leq(A ; B)(Y)=\mathrm{id}$. Thus $A ; B$ is a POVM.

Example 7. We apply the above construction to the spin example from [14]. Consider a system consisting of one spin- $\frac{1}{2}$ particle, modeled as the Hilbert space $\mathbb{C}^{2}$. The direction of the spin has a value in the unit sphere $\mathbb{S}^{2}$, and is given by the POVM

$$
\begin{gathered}
D: \Sigma_{\mathbb{S}^{2}} \rightarrow \mathscr{E} f\left(\mathbb{C}^{2}\right) \\
D(M)=\frac{1}{4 \pi} \int_{M}(\mathrm{id}+\vec{n} \cdot \vec{\sigma}) \mathrm{d} \vec{n}
\end{gathered}
$$

Here $\mathrm{d} \vec{n}$ is the usual measure on the unit sphere, and $\vec{\sigma}=\left(\sigma_{x}, \sigma_{y}, \sigma_{z}\right)$ is the vector consisting of the Pauli matrices:

$$
\sigma_{x}=\left(\begin{array}{cc}
0 & 1 \\
1 & 0
\end{array}\right), \quad \sigma_{y}=\left(\begin{array}{cc}
0 & -i \\
i & 0
\end{array}\right), \quad \sigma_{z}=\left(\begin{array}{cc}
1 & 0 \\
0 & -1
\end{array}\right) .
$$

If we pick a direction $\vec{n} \in \mathbb{S}^{2}$, then we can also measure the spin component along the direction $\vec{n}$. This measurement has two possible outcomes, which we label by + and - . The corresponding POVM is $S_{\vec{n}}: \Sigma_{\{ \pm\}} \rightarrow \mathscr{E} f\left(\mathbb{C}^{2}\right)$, defined by $S_{\vec{n}}(\{ \pm\})=\frac{1}{2}(\mathrm{id} \pm \vec{n} \cdot \vec{\sigma})$. Physically, the probability that the outcome is + indicates how close the actual spin direction of the particle is to $\vec{n}$.

We perform the following experiment on the system. First we measure the spin direction, which has outcome $\vec{n}$. Then we measure the spin component along this direction, i.e. we perform the measurement $S_{\vec{n}}$. Since the spin direction of the particle is in this situation equal to the measurement direction, we expect that the second measurement always gives outcome + . The outcome space of the composite 
measurement $D ; S$ is $\bigcup_{\vec{n} \in \mathbb{S}^{2}}\{ \pm\} \cong \mathbb{S}^{2} \times\{ \pm\}$. According to the physical interpretation, this composite measurement is determined by

$$
\begin{gathered}
(D ; S)(M \times\{-\})=0 \\
(D ; S)(M \times\{+\})=D(M)
\end{gathered}
$$

We can also verify this using the sequential composition formula. From the definition of the POVM $D$ it is immediate that its Radon-Nikodym derivative is

$$
\frac{\mathrm{d} D}{\mathrm{~d} \vec{n}}(\vec{n})=\frac{1}{4 \pi}(\mathrm{id}+\vec{n} \cdot \vec{\sigma}) .
$$

Then the 'minus' case of the sequential composition formula becomes:

$$
\begin{aligned}
(D ; S)(M \times\{-\}) & =\int_{M} \sqrt{\frac{\mathrm{d} D}{\mathrm{~d}}(\vec{n})} S_{\vec{n}}(\{-\}) \sqrt{\frac{\mathrm{d} D}{\mathrm{~d}}(\vec{n})} \mathrm{d} \vec{n} \\
& =\frac{1}{8 \pi} \int_{M}(\sqrt{\mathrm{id}+\vec{n} \cdot \vec{\sigma}}(\mathrm{id}-\vec{n} \cdot \vec{\sigma}) \sqrt{\mathrm{id}+\vec{n} \cdot \vec{\sigma}}) \mathrm{d} \vec{n} \\
& =\frac{1}{8 \pi} \int_{M}(\mathrm{id}+\vec{n} \cdot \vec{\sigma})(\mathrm{id}-\vec{n} \cdot \vec{\sigma}) \mathrm{d} \vec{n} \\
& =\frac{1}{8 \pi} \int_{M}\left(\mathrm{id}-(\vec{n} \cdot \vec{\sigma})^{2}\right) \mathrm{d} \vec{n}
\end{aligned}
$$

For the third equality sign, we used that a square root $\sqrt{A}$ commutes with every operator that commutes with $A$. A well-known property of the Pauli matrices is that $(\vec{n} \cdot \vec{\sigma})^{2}=\mathrm{id}$ for each unit vector $\vec{n}$. From this it follows that $(D ; S)(M \times\{-\})=0$. An analogous computation shows that $(D ; S)(M \times\{+\})=D(M)$.

\section{Characterization of continuous POVMs}

In Section 4 we saw that we need continuity conditions on POVMs in order to define sequential composition. Therefore we will now study continuous POVMs in more detail and provide a few equivalent characterizations. It will turn out that in the continuous case von Neumann algebras form a more natural setting than effect algebras. Our main examples of von Neumann algebras are constructed from Hilbert spaces and measure spaces. If $H$ is a Hilbert space, then $\mathscr{B}(H)$ will denote the von Neumann algebra of bounded linear operators on $H$. Recall that a measure space is a measurable space together with a measure. For a measure space $(X, \mu)$, let $L^{\infty}(X, \mu)$ be the algebra of $\mu$-essentially bounded functions from $X$ to $\mathbb{C}$, modulo equality almost everywhere. We will assume throughout this section that $X$ arises from a compact Hausdorff space and that $\mu(X)$ is finite.

The duality for non-continuous POVMs boiled down to the duality between states and effects. For continuous POVMs we will replace this by the interplay between a von Neumann algebra and its normal states, or its predual. To describe this in more detail, we will use several categories of von Neumann algebras. The standard notion of morphism between $\mathrm{C}^{*}$-algebras is a $*$-homomorphism, which is a bounded linear map preserving multiplication, unit, and involution. For von Neumann algebras we usually impose an additional condition: a map between von Neumann algebras is called normal if it preserves joins of countable increasing chains. This is equivalent to preservation of countable sums of orthogonal projections, see e.g. [19] for details. The category of unital von Neumann algebras with normal *-homomorphisms will be denoted $\mathbf{v N}$. Sometimes it is more appropriate to use a weaker notion of morphism. The category with von Neumann algebras as objects and normal linear maps preserving positivity and the unit as morphisms is denoted $\mathbf{v N} \mathbf{P U}$.

The predual $A_{\#}$ of a von Neumann algebra $A$ consists of all normal linear functionals from $A$ to $\mathbb{C}$. If $A$ is unital, then the predual is equipped with a canonical trace map $\tau: A_{\#} \rightarrow \mathbb{C}$, given by evaluation 
at the unit. For example, the predual of $\mathscr{B}(H)$ is the collection of trace-class operators $\mathscr{T}(H)$, and the canonical trace map is the ordinary trace $\operatorname{tr}: \mathscr{T}(H) \rightarrow \mathbb{C}$. The predual of $L^{\infty}(X, \mu)$ is $L^{1}(X, \mu)$, i.e. the measurable functions $f: X \rightarrow \mathbb{C}$ such that the integral $\int_{X}|f| \mathrm{d} \mu$ is finite. In this case, the trace map is integration $\int_{X}(-) \mathrm{d} \mu$.

The structure of a predual can be captured abstractly by base norm spaces, see e.g. [1, 21]. Let $V$ be an ordered vector space, and $\tau: V \rightarrow \mathbb{C}$ a positive linear functional. A convex subset $C$ of $V$ is called linearly bounded if $C \cap L$ is bounded for every line $L$ through the origin. Let $K=\tau^{-1}(1) \subseteq V$; the pair $(V, \tau)$ is said to be a base norm space if the convex hull of $K \cup-K$ is linearly bounded. Base norm spaces form a category BNS in which a morphism from $(V, \tau)$ to $\left(V^{\prime}, \tau^{\prime}\right)$ is a positive linear map $f: V \rightarrow V^{\prime}$ for which $\tau^{\prime} \circ f=\tau$.

The following result shows how to view continuous POVMs as morphisms between von Neumann algebras.

Proposition 8. There is a bijective correspondence between:

- POVMs $\Sigma_{X} \rightarrow \mathscr{E} f(H)$ that are $\mu$-continuous and have bounded variation;

- Normal positive unital maps $L^{\infty}(X, \mu) \rightarrow \mathscr{B}(H)$.

Proof. Let $\varphi: \Sigma_{X} \rightarrow \mathscr{E} f(H)$ be a POVM. Define a map $\psi_{\varphi}: L^{\infty}(X, \mu) \rightarrow \mathscr{B}(H)$ by $\psi_{\varphi}(f)=\int_{X} f \mathrm{~d} \varphi$. This integral is well-defined since $f$ is essentially bounded. To verify that the map $\psi_{\varphi}$ is well-defined, we have to check that it maps functions that are zero almost everywhere to the zero operator. If an indicator function $\mathbf{1}_{M}$ is zero almost everywhere, then $\mu(M)=0$, so from $\mu$-continuity of $\varphi$ it follows that $\int \mathbf{1}_{M} \mathrm{~d} \varphi=\varphi(M)=0$. For general functions in $L^{\infty}(X, \mu)$ this follows from linearity and continuity of the integral. Furthermore the map $\psi_{\varphi}$ is positive and unital. It preserves joins of countable chains since $\varphi$ is a POVM. Every positive map between von Neumann algebras is bounded, see [20, Prop. 1.3.7] for a proof.

In the other direction, given a map $\psi: L^{\infty}(X, \mu) \rightarrow \mathscr{B}(H)$, define $\varphi_{\psi}: \Sigma_{X} \rightarrow \mathscr{E} f(H)$ by $\varphi_{\psi}(M)=$ $\psi\left(\mathbf{1}_{M}\right)$. Then $\varphi_{\psi}(M)$ is positive because $\psi$ preserves positivity, and $\varphi_{\psi}(M) \leq \psi\left(\mathbf{1}_{X}\right)=\mathrm{id}$, so $\varphi_{\psi}(M)$ is an effect. The map $\varphi_{\psi}$ is a morphism of $\sigma$-effect algebras since $\psi$ is linear, normal, and unital. To establish $\mu$-continuity of $\varphi_{\psi}$, suppose that $\mu(M)=0$. Then $\mathbf{1}_{M}$ is zero almost everywhere, hence $\varphi_{\psi}(M)=\psi\left(\mathbf{1}_{M}\right)=\psi(0)=0$. Finally, $\varphi_{\psi}$ has bounded variation because

$$
\sup \sum_{i}\left\|\varphi_{\psi}\left(X_{i}\right)\right\|=\sup \sum_{i}\left\|\psi\left(\mathbf{1}_{X_{i}}\right)\right\| \leq \sup \sum_{i}\|\psi\| \mu\left(X_{i}\right)=\|\psi\| \mu(X)<\infty .
$$

It is easy to see that both constructions are inverses.

Observe that the construction of the map between von Neumann algebras from a POVM did not use the fact that the POVM has bounded variation. Thus we obtain the following consequence.

Corollary 9. Every $\mu$-continuous POVM has bounded variation.

Therefore we can simply work with $\mu$-continuous POVMs from now on, ignoring the condition on the variation. It is also possible to characterize projection-valued measures as maps between von Neumann algebras, by restricting the above correspondence.

Corollary 10. There is a bijective correspondence between:

- PVMs $\Sigma_{X} \rightarrow \mathscr{P} \operatorname{roj}(H)$ that are $\mu$-continuous;

- Normal $*$-homomorphisms $L^{\infty}(X, \mu) \rightarrow \mathscr{B}(H)$. 
Proof. Let $\psi: L^{\infty}(X, \mu) \rightarrow \mathscr{B}(H)$ be a normal $*$-homomorphism. By Proposition 8 , it gives a $\mu$ continuous POVM $\varphi: \Sigma_{X} \rightarrow \mathscr{E} f(H)$. We have to check that each $\varphi(M)$ is a projection:

$$
\varphi(M)^{2}=\psi\left(\mathbf{1}_{M}\right)^{2}=\psi\left(\left(\mathbf{1}_{M}\right)^{2}\right)=\psi\left(\mathbf{1}_{M}\right)=\varphi(M)
$$

where we used that $\psi$ preserves multiplication in the second equality sign.

Conversely, a $\mu$-continuous PVM $\varphi$ gives a normal positive unital map $\psi: L^{\infty}(X, \mu) \rightarrow \mathscr{B}(H)$. To show that $\psi$ preserves multiplication, we start by considering indicator functions:

$$
\psi\left(\mathbf{1}_{M} \mathbf{1}_{N}\right)=\psi\left(\mathbf{1}_{M \cap N}\right)=\varphi(M \cap N)=\varphi(M) \varphi(N)=\psi\left(\mathbf{1}_{M}\right) \psi\left(\mathbf{1}_{N}\right)
$$

The third equality sign is a property that characterizes the projection-valued measures. All essentially bounded functions from $X$ to $\mathbb{C}$ can be written as a countable join of sums of indicator functions, modulo equality almost everywhere. Since $\psi$ preserves sums and countable joins, it follows that $\psi(f g)=\psi(f) \psi(g)$ for all $f$ and $g$.

The characterization of continuous POVMs as maps between the von Neumann algebras $L^{\infty}(X, \mu)$ and $\mathscr{B}(H)$ is in line with the Heisenberg picture of quantum mechanics. There is also a characterization from the Schrödinger point of view, analogous to considering POVMs as maps between $\mathscr{G}$-algebras.

Proposition 11. There is a bijective correspondence between:

- Normal positive unital maps $L^{\infty}(X, \mu) \rightarrow \mathscr{B}(H)$;

- Maps $\mathscr{T}(H) \rightarrow L^{1}(X, \mu)$ of base norm spaces.

Proof. Let $\varphi: L^{\infty}(X, \mu) \rightarrow \mathscr{B}(H)$ be normal positive unital. The predual $L^{\infty}(X, \mu)_{\#}$ of $L^{\infty}(X, \mu)$ is isomorphic to $L^{1}(X, \mu)$, so to define a map $\mathscr{T}(H) \rightarrow L^{1}(X, \mu)$, we can also define a map $\Phi_{\varphi}$ from $\mathscr{T}(H)$ into the normal functionals on $L^{\infty}(X, \mu)$. For this we take $\Phi_{\varphi}(T)(f)=\operatorname{tr}(T \varphi(f))$. The assignment $f \mapsto \operatorname{tr}(T \varphi(f))$ lies in $L^{\infty}(X, \mu)_{\#}$, because $\varphi$ is normal. The map $\Phi_{\varphi}$ is positive since $\varphi$ is. To check that $\Phi_{\varphi}$ commutes with the maps into $\mathbb{C}$, use that the integration map $\int_{X}(-) \mathrm{d} \mu: L^{\infty}(X, \mu) \rightarrow \mathbb{C}$ corresponds to the map $L^{\infty}(X, \mu)_{\#} \rightarrow \mathbb{C}$ given by $\psi \mapsto \psi(1)$. From this it follows that $\Phi_{\varphi}$ is a map of base norm spaces.

Now we will show how to assign a map $L^{\infty}(X, \mu) \rightarrow \mathscr{B}(H)$ to a map $\Phi: \mathscr{T}(H) \rightarrow L^{1}(X, \mu)$. First define a map $\varphi_{\Phi}: L^{\infty}(X, \mu) \rightarrow \mathscr{T}(H)^{*}$ by $\varphi_{\Phi}(f)(T)=\int_{X} f(x) \Phi(T)(x) \mathrm{d} x$. This integral exists since $f$ is bounded and $\Phi(T)$ is integrable. Since the dual of the Banach space $\mathscr{T}(H)$ is isomorphic to the space of bounded operators on $H$, this gives a map $L^{\infty}(X, \mu) \rightarrow \mathscr{B}(H)$, also denoted $\varphi_{\Phi}$. Positivity of $\varphi_{\Phi}$ follows from positivity of $\Phi$. To show that $\varphi_{\Phi}$ is unital, note that the unit of $L^{\infty}(X, \mu)$ is the constant function with value 1 , and the unit of $\mathscr{T}(H)^{*}$ is the trace. Then unitality of $\varphi_{\Phi}$ follows since $\Phi$ is a morphism of base norm spaces:

$$
\varphi_{\Phi}(1)(T)=\int_{X} \Phi(T)(x) \mathrm{d} x=\operatorname{tr}(T) .
$$

The map $\varphi_{\Phi}$ is normal because integrals are continuous. The constructions above are clearly inverses.

Again we can rephrase the duality result above as an equivalence between comma categories. First 
we will establish the following diagram of categories and functors.

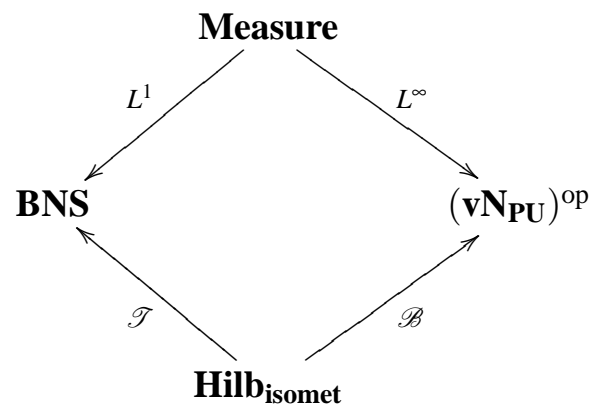

The functors $\mathscr{T}$ and $\mathscr{B}$ act on morphisms via conjugation. Formally, $\mathscr{B}(f)(A)=f^{\dagger} A f$ and $\mathscr{T}(f)(T)=$ $f T f^{\dagger}$.

The category Measure has measure spaces as objects. A morphism from $(X, \mu)$ to $(Y, v)$ is a measurable map $f: X \rightarrow Y$ such that the measure $\mu \circ f^{-1}$ on $Y$ is $v$-continuous, in other words, $v(N)=0$ implies $\mu\left(f^{-1}[N]\right)=0$. We have seen the action of $L^{\infty}$ and $L^{1}$ on objects before. Let $f:(X, \mu) \rightarrow(Y, v)$ be a morphism in Measure. Define $L^{\infty}(f): L^{\infty}(Y, v) \rightarrow L^{\infty}(X, \mu)$ by $L^{\infty}(f)(\varphi)=\varphi \circ f$. If $\varphi$ is $v$-essentially bounded, then $\varphi \circ f$ is $\mu$-essentially bounded because $\mu \circ f^{-1}$ is $v$-continuous. It is clear that $L^{\infty}(f)$ is a morphism in $\mathbf{v} \mathbf{N}_{\mathbf{P U}}$. To define $L^{1}(f)(\varphi)$ for $\varphi \in L^{1}(X, \mu)$, we first introduce a new measure $\lambda$ on $Y$ via $\lambda(N)=\int_{f^{-1}[N]} \varphi \mathrm{d} \mu$. This $\lambda$ is $v$-continuous, so we can define $L^{1}(f)(\varphi)$ to be its derivative $\frac{\mathrm{d} \lambda}{\mathrm{d} \nu}$. Thus $L^{1}(f)(\varphi)$ is the unique function satisfying $\int_{N} L^{1}(f)(\varphi) \mathrm{d} \nu=\int_{f^{-1}[N]} \varphi \mathrm{d} \mu$. Clearly $L^{1}(f)(\varphi)$ is integrable, and $L^{1}(f)$ is a morphism in BNS.

Corollary 12. The categories $\left(\mathscr{T} \downarrow L^{1}\right)$ and $\left(\mathscr{B} \downarrow L^{\infty}\right)$ are equivalent.

Proof. On objects, this was established in Proposition 11. On morphisms, this amounts to proving naturality of the correspondence in the Proposition. Pick any isometry $f: H \rightarrow K$ and let $\varphi: L^{\infty}(X, \mu) \rightarrow$ $\mathscr{B}(K)$ be a normal positive unital map. Then we have to show that $\Phi_{\mathscr{B}(f) \circ \varphi}=\Phi_{\varphi} \circ \mathscr{T}(f)$. This holds because

$$
\Phi_{\mathscr{B}(f) \circ \varphi}(T)(g)=\operatorname{tr}\left(T f^{\dagger} \varphi(g) f\right)=\operatorname{tr}\left(f T f^{\dagger} \varphi(g)\right)=\left(\Phi_{\varphi} \circ \mathscr{T}(f)\right)(T)(g) .
$$

Finally we have to prove that $\Phi_{\varphi \circ L^{\infty}(f)}(T)=\left(L^{1}(f) \circ \Phi_{\varphi}\right)(T)$ for $f:(X, \mu) \rightarrow(Y, v)$. This is equivalent to showing that the integrals $\int_{N} \Phi_{\varphi \circ L^{\infty}(f)}(T) \mathrm{d} \nu$ and $\int_{N}\left(L^{1}(f) \circ \Phi_{\varphi}\right)(T) \mathrm{d} v$ are equal for each $N$. If we identify elements of $L^{1}(Y)$ with normal functionals on $L^{\infty}(Y)$, then integration over $N$ amounts to plugging in the functional $\mathbf{1}_{N}$. Hence the first integral is equal to $\operatorname{tr}\left(T \varphi\left(\mathbf{1}_{N} \circ f\right)\right)$, and the second integral is equal to $\operatorname{tr}\left(T \varphi\left(\mathbf{1}_{f^{-1}[N]}\right)\right)$, thus the integrals are the same.

\section{Conclusion}

We have established bijective correspondences between the following representations of POVMs:

- Morphisms of $\sigma$-effect algebras $\Sigma_{X} \rightarrow \mathscr{E} f(H)$;

- Morphisms of $\sigma$-effect modules $\operatorname{Meas}(X,[0,1]) \rightarrow \mathscr{E} f(H)$;

- Morphisms of $\mathscr{G}$-algebras $\mathscr{D} \mathscr{M}(H) \rightarrow \mathscr{G}(X)$.

In the situation where the space $X$ is compact and equipped with a finite measure $\mu$, we obtain correspondences between the following: 
- POVMs $\Sigma_{X} \rightarrow \mathscr{E} f(H)$ that are $\mu$-continuous;

- Normal positive unital maps $L^{\infty}(X, \mu) \rightarrow \mathscr{B}(H)$;

- Maps $\mathscr{T}(H) \rightarrow L^{1}(X, \mu)$ of base norm spaces.

These correspondences can be phrased as equivalences between comma categories. The object part of these equivalences gives the bijective correspondences above, and since we have shown that there is also an equivalence between the morphisms of the comma categories, the above correspondences are natural.

Many POVMs occuring in physics are covariant with respect to a symmetry group or groupoid, as discussed in [20, 22]. For future research, it would be interesting to see how our results can be extended to the covariant setting using convolution algebras. Another possible direction would be to study the sequential composition for POVMs in more detail, for example by finding an axiomatization generalizing the one for effects in [11].

Acknowledgements. This research has been financially supported by the Netherlands Organisation for Scientific Research (NWO) under TOP-GO grant no. 613.001.013 (The logic of composite quantum systems). Thanks are due to Robert Furber and Bart Jacobs for helpful discussions and comments.

\section{References}

[1] E. Alfsen (1971): Compact Convex Sets and Boundary Integrals. Springer-Verlag, doi: $10.1007 / 978-3-642-65009-3$

[2] George D. Birkhoff \& John von Neumann (1936): The logic of quantum mechanics. Annals of Math. 37, pp. $823-834$, doi $10.2307 / 1968621$.

[3] P. Busch (2003): Quantum states and generalized observables: a simple proof of Gleason's theorem. Phys. Rev. Lett. 91(12), doi 10.1103/PhysRevLett.91.120403

[4] E. D’Hondt \& P. Panangaden (2006): Quantum weakest preconditions. Math. Struct. in Comp. Sci. 16(3), pp. 429-451, doi:10.1017/S0960129506005251

[5] J. Diestel \& J. Uhl (1977): Vector Measures. American Mathematical Society, doi $10.1090 /$ surv/015.

[6] A. Dvurečenskij \& S. Pulmannová (2000): New Trends in Quantum Structures. Kluwer Acad. Publ., Dordrecht, doi $10.1007 / 978-94-017-2422-7$.

[7] D. J. Foulis \& M.K. Bennett (1994): Effect Algebras and Unsharp Quantum Logics. Found. Phys. 24(10), pp. 1331-1352, doi $10.1007 / \mathrm{BF} 02283036$

[8] M. Giry (1982): A categorical approach to probability theory. In B. Banaschewski, editor: Categorical Aspects of Topology and Analysis, Lecture Notes in Mathematics 915, Springer Berlin Heidelberg, pp. 6885, doi:10.1007/BFb0092872

[9] S. Gudder (1998): Morphisms, tensor products and $\sigma$-effect algebras. Rep. Math. Phys. 42(3), pp. 321-346, doi: $10.1016 /$ S0034-4877(99)80003-2.

[10] S. Gudder (2010): Sequential product of quantum effects: an overview. Int. J. Math. Phys. 49, pp. 3118-3130, doi:10.1007/s10773-010-0390-6

[11] S. Gudder \& R. Greechie (2002): Sequential products on effect algebras. Rep. Math. Phys. 49, pp. 87-111, doi:10.1016/S0034-4877(02)80007-6

[12] S. Gudder \& G. Nagy (2001): Sequential quantum measurements. J. Math. Phys. 42(11), pp. 5212-5222, doi: $10.1063 / 1.1407837$.

[13] S. Gudder \& S. Pulmannová (1998): Representation theorem for convex effect algebras. Commentationes Mathematicae Universitatis Carolinae 39(4), pp. 645-659. 
[14] T. Heinosaari \& M. Ziman (2012): The Mathematical Language of Quantum Theory. From Uncertainty to Entanglement. Cambridge Univ. Press.

[15] B. Jacobs (2012): New directions in categorical logic, for classical, probabilistic, and quantum logic. http://arxiv.org/abs/1205.3940

[16] B. Jacobs (2013): Measurable Spaces and their Effect Logic. Logic in Computer Science (LICS 2013).

[17] B. Jacobs \& J. Mandemaker (2012): The Expectation Monad in Quantum Foundations. In B. Jacobs, P. Selinger \& B. Spitters, editors: Quantum Physics and Logic (QPL) 2011, Elect. Proc. in Theor. Comp. Sci. 95, pp. 143-182.

[18] B. Jacobs \& J. Mandemaker (2012): Relating Operator Spaces via Adjunctions. In J. Chubb Reimann, V. Harizanov \& A. Eskandarian, editors: Logic and Algebraic Structures in Quantum Computing and Information, Lect. Notes in Logic, Cambridge Univ. Press. See arxiv.org/abs/1201.1272.

[19] R. Kadison \& J. Ringrose (1983): Fundamentals of the Theory of Operator Algebras. Academic Press.

[20] N. Landsman (1998): Mathematical Topics Between Classical and Quantum Mechanics. Springer, doi: $10.1007 / 978-1-4612-1680-3$

[21] R. Nagel (1974): Order unit and base norm spaces. In A. Hartkämper \& H. Neumann, editors: Foundations of Quantum Mechanics and Ordered Linear Spaces, Lecture Notes in Physics 29, Springer Berlin Heidelberg, pp. 23-29, doi $10.1007 / 3-540-06725-6-4$

[22] F. Schroeck (1996): Quantum Mechanics on Phase Space. Kluwer Acad. Publ., doi:10.1007/978-94-017-2830-0 